\title{
TITEL
}

\section{Jasmin Siri \\ Die prekäre Position der Opfer des NSU. Bericht aus einer laufenden öffentlichen Verhandlung}

\section{A. Einfübrung}

\begin{abstract}
"Das ist ein gutes Deutschland, das beste, das wir jemals hatten." (Joachim Gauck in einer Rede zur Münchner Sicherbeitskonferenz am 31.1.2014)

„,Döner-Killer', wie der Mörder von Türken inzwischen auch genannt wird. “ (AZ vom 15.08.2006)
\end{abstract}

Nicht nur vor dem Münchner Oberlandesgericht wird gerade über den NSU verhandelt. In den Massenmedien und an öffentlichen Orten wie Theatern, Ausstellungen, Reden und Büchern wird ebenfalls verhandelt; wenn auch weniger der Fall selbst als seine Bedeutung und die verschiedenen Rollen, die den Fall umspannen. Es wird über Verantwortung, Täterschaft und Opferschaft verhandelt und irgendwann wird in dieser diskursiven Konstellation auch mehr oder weniger festgeschrieben werden, was da eigentlich passiert ist im der Selbstbeschreibung nach von den Spuren des Nazismus gereinigten, kosmopolitischen, „guten“ (Gauck) Deutschland.

Wie passen die über viele Jahre nicht verfolgten rassistischen Morde zu dieser offiziellen Selbstbeschreibung als gutem Deutschland, das sich nun sogar qua präsidial-theologischer Sprachregelung von seiner dunklen Vergangenheit geläutert sehen darf? Wie passt es zum guten Deutschland, dass Witwen und Kinder der vom NSU ermordeten Menschen durch die Ermittlung über Jahre hin kriminalisiert wurden? Wie passt hierzu, dass Behörden Ermittlungsergebnisse nicht berücksichtigt oder Ermittlungen sogar behindert haben? Welche Konsequenzen haben derlei Widersprüche zwischen Anspruch/ Selbstbeschreibung und Wirklichkeit, zwischen versagenden Ermittlungsbehörden und Nazimorden auf der einen und der Selbstdarstellung des guten, geläuterten Deutschlands auf der anderen Seite für mediale und öffentliche Debatten?

Im Folgenden werde ich keinen repräsentativen Überblick über die mediale Debatte zum NSU in der BRD vorstellen. Die Beispiele und interpretativen Schlussfolgerungen sind aber auch nicht willkürlich gewählt und basieren auf einer reichhaltigen Datensammlung. So beziehe ich mich erstens auf Material, das aus einer umfassenden Erhebung der medialen Darstellung der NSU-Morde und der NSU-TerroristInnen in Deutschland, 
der Türkei und Großbritannien basieren ${ }^{1}$ sowie zweitens auf einen Sammelband zum Thema, der 2013 erschienen ist. ${ }^{2}$ Die Vorarbeiten zu diesem Band wie auch die Ergebnisse einer kleinen Studie zur medialen Verhandlung von Gender im Kontext der Berichterstattung zum Prozess ${ }^{3}$ fließen ebenfalls in diesen Text ein.

Ich möchte argumentieren, dass sich bereits heute - trotz aller Vorläufigkeit dieses Berichtes angesichts des laufenden Prozesses und der vielen Aktivitäten und Kommentare, die ihn begleiten - recht stabile Muster der Narration und Deutung der NSU-Verbrechen herausarbeiten lassen. Auch zeigen sich Muster der Entstehung und Anordnung verschiedener „Rollen“, die in der Erklärung und Deutung dieser Verbrechen eine Rolle spielen. Besonders interessant ist hierbei die Frage, wie TäterInnen und Opfer(-angehörige) adressiert und medial konzipiert werden. In diesem Text will ich den Focus auf die prekäre Position der Opfer des NSU und der Opferangehörigen in der medialen Öffentlichkeit legen. Es soll danach gefragt werden, wie Opfer und Angehörige beschrieben und adressiert werden. Es wird sich zeigen, dass es mitnichten so ist, dass die Ermordung einer Person automatisch zur Anerkennung der Trauer um diese Person führt. So basiert ein guter Teil der öffentlichen Empörung über die Vorgänge der letzten vierzehn Jahre auf der Tatsache, dass nicht nur unzureichend und schlecht ermittelt worden war, sondern die Opfer und ihre Familien ohne ausreichenden Verdacht über Jahre als potentielle Täter behandelt wurden. Zeitungen und Ermittlungsakten waren voll der Unterstellungen und fantastischer Ideen über kriminelle „Parallelgesellschaften“. Die „Dönermorde“ - so die folkloristisch-rassistische Bezeichnung der Morde nicht nur in Boulevardmedien - wären sicher Ergebnis von Kämpfen der „Türkenmafia“ - oder derselben gegen die „Russenmafia“, die Opfer seien wohl Drogenhändler, Zuhälter oder anderweitig halbweltlich engagiert. Rassistische Stereotype blühten bei Medien und Ermittlungsbehörden, die Namensgebung der „SOKO Bosporus“ ist nur ein (harmloseres) von vielen Beispielen. Alleine, dass die Opfer nicht deutscher Abstammung waren, reichte scheinbar, um von sogenannter "Ausländerkriminalität“ auszugehen.

Ermordet zu werden bedeutet also noch lange nicht, in der Öffentlichkeit auch als ein „echtes Opfer" wahrgenommen und betrauert zu werden. Hiervon handelt mittels einer empirischen und theoretischen Auseinandersetzung das zweite Kapitel. Im Laufe der Prozessberichterstattung und der öffentlichen Debatte tauchten außerdem auch andere

1 Die Ergebnisse der noch nicht abgeschlossenen Studie werden (voraussichtlich noch 2014) in englischer, deutscher und türkischer Sprache publiziert werden. Denli E Siri (forthcoming): „Medial Representations of NSU-Terrorism in Germany, Turkey and Great Britain“, bzw. „Die mediale Darstellung des NSU-Terrorismus in Deutschland, der Türkei und Großbritannien“. Ich beziehe mich außerdem auf Vorarbeiten zu dem Buch „NSU-Terror. Ermittlungen am rechten Abgrund“ (gem. mit Schmincke herausgegeben) und Vorbereitungen zur Veranstaltung „Wenn aus Menschen „Döner’ werden. Soziologische Analysen eines politischen Diskurses“ auf dem Kongress der Deutschen Gesellschaft für Soziologie in Bochum 2012 gem. mit Villa, Lessenich und Schmincke.

2 Schmincke E Siri (Hg.) 2013.

3 Die gemeinsam mit Villa formulierten Gedanken wurden am15.3.14 auf der 21st Conference des Council for European Studies in Washington D.C. vorgetragen (Titel: Terror-Bride and Hooligan. An Analysis of the Gendered Discourse Regarding Right-Wing Terrorism in Germany).

NK 26. Jg. 2/2014 
potentielle Opfer auf, wie zum Beispiel die Bundesrepublik Deutschland und ihre Integrität, aber auch die attackierten Geheimdienste und die Familien der Haupttäter und der Haupttäterin. Im dritten Kapitel werde ich daher diskutieren, ob und inwiefern die verschiedenen vorgestellten „Rollen“ und Opferpositionen im Diskurs über die Verbrechen des NSU miteinander in Konkurrenz um öffentliche Aufmerksamkeit stehen.

\section{B. Wenn aus Opfern Täter werden... Der Ausschluss aus dem Opferstatus ${ }^{4}$}

"In dieser Ehe, bohrte der Polizist weiter, habe es offenbar Heimlichkeiten, Unehrlichkeiten, Unklarbeiten in Geldangelegenheiten gegeben, so gut könne diese Ehe ja wobl doch nicht gewesen sein.

Doch, antwortete meine Mutter, das war sie!

Und hatte er eine Freundin?

Nein. Nein!

Vielleicht, sagte meine Mutter dann müde, vielleicht vor dem Hadsch. Womöglich vor der Pilgerreise nach Mekka, das wäre die einzige denkbare Zeit, womöglich damals. Aber nicht danach.

Aha, hakte der Polizist ein: Sie geben also zu, dass Ihr Mann vielleicht ein Verbältnis hatte. Wie heißt die Fran?

Nein, sagte meine Mutter verzweifelt, nein, so was das nicht gemeint!"s

In den Jahren 2000-2007 ermordeten NSU-Terroristen 10 Menschen und verletzten viele weitere schwer an Körpern und Seelen. Dies blieb bekanntermaßen bis zur Selbstenttarnung der TerroristInnen im Jahre 2011 im Dunklen. Die Namen der Opfer der rassistischen Mordserie lauten Enver Şimşek, Abdurrahim Özüdoğru, Süleyman Taşköprü, Habil Kılıç, Mehmet Turgut, İsmail Yaşar, Theodoros Boulgarides, Mehmet Kubaşık und Halil Yozgat. Dass die Mörder sie als Migranten einordneten, war der einzige Grund für ihre Ermordung. Auch die Polizistin Michéle Kiesewetter war ein Opfer des NSU, allerdings vermuteten die Ermittler hier keinen Zusammenhang, sondern suchten den Täter unter Sinti und Roma, die sich in der Nähe des Tatorts aufgehalten haben sollten. Auch ein Kollege von Frau Kiesewetter wurde lebensgefährlich verletzt.

Ein rechtsradikaler Hintergrund der Mordserie wurde über die Jahre mehrfach ausgeschlossen, zwei Fallanalysen bayerischer Fahnder und des FBI, die diesen Zusammenhang herstellten, wurden ignoriert. ${ }^{6}$ Die Reihe der Ermittlungsfehler verschiedener Behörden im Zusammenspiel föderalistischer Konkurrenzen ist so lang wie die Liste der traurigen Absurditäten und die Vertuschungen, die die Ermittlungen begleiten. Verwiesen sei hier beispielhaft auf die Hinzuziehung eines iranischen Geisterbeschwörers durch die Hamburger Polizei im Jahre 2006 und auf die „Aktion Konfetti“, der Vernichtung

4 Die Ausführungen in diesem Kapitel beruhen auf dem Aufsatz „Vermisst? Zur Entstehung von Positionen der Unsichtbarkeit.“ im Band „NSU-Terror. Ermittlungen am rechten Abgrund“, S. 193-202.

5 Simssek 2013, 118.

6 Kleffner 2013, $32 \mathrm{ff}$. 
von inzwischen teilweise rekonstruierten Akten über V-Leute im rechten Spektrum durch den Verfassungsschutz in den Jahren 2011 und 2012. ${ }^{7}$

Die traumatischen Erlebnisse der Familien der Ermordeten, ihre Kriminalisierung und Isolation, haben Angehörige und OpferanwältInnen seit dem Beginn des Prozesses immer wieder beschrieben. Semiya Şimşek, Tochter des vom NSU ermordeten Blumenhändlers Enver Şimşek, schildert in ihrem autobiografischen Buch „Schmerzliche Heimat" die Leiden ihrer Familie. ${ }^{8}$ Nicht nur die Şimşeks, auch andere Hinterbliebenen wurden nicht geschont oder gar getröstet, sondern vielmehr unter Druck gesetzt, ausgeforscht und kriminalisiert. Neben den Verlust einer geliebten Person trat so soziale Ächtung und Isolation, wie mehrere Opferangehörige beschrieben haben. Selbst vor grausamen Tricks schreckten Ermittler nicht zurück: Mehreren Witwen, so berichtet Heike Kleffner, wurden Fotos einer blonden Frau gezeigt, die angeblich die Geliebte der Ehemänner gewesen sei. ${ }^{9}$ Aus Opfern wurden Täter. „Die Tatsache, dass die unter Verdacht stehenden Communities keine brauchbaren Hinweise auf mögliche Täter lieferten, begründeten leitende Ermittler dann mit der Existenz eines ,milieutypischen Schweigekartells"“. 10

Der Frage danach, wer ein betrauernswertes Opfer ist, verdeutlicht, dass moderne Menschen in Demokratien zwar grundsätzlich in alle Systeme der Gesellschaft wie dem Rechtssystem, Erziehungssystem und dem politischen System integriert sind ${ }^{11}$ damit aber noch im Dunklen bleibt, welchen Status und welche Art der Anerkennung ihrer Sprecherposition sie innerhalb dieser Systeme besitzen. Die Philosophin Judith Butler nutzt daher die Frage danach, um wen in einer Gesellschaft getrauert wird, als Lackmustest für ihre Offenheit und gezielte wie unbewusste Exklusionen. Die Frage, ,Um wen trauern wir öffentlich?' macht Exklusionen an den „Rändern“ von Kollektiven mehr als deutlich. ${ }^{1213}$

Die Geschichte der Opfer des NSU zeigt, wie voraussetzungsreich die Konstruktion dessen ist, was wir Rechtssubjekt oder Individuum nennen. Die Subjekte sind nicht einfach schon immer da und schon gar nicht wird ein jedes menschliche Lebewesen gleichermaßen als solches erkannt und anerkannt. ${ }^{14}$ Der mediale Diskurs um den NSU und seine Opfer macht deutlich, dass auch im modernen Rechtsstaat einige gleicher sind als andere, besonders wenn die Wertung „Ausländer“ oder „Türke“ ins Spiel kommt. Die Schilderungen von Opferfamilien über den Verlust sozialer und beruflicher Kontakte

7 Ebd. 38.

8 Simsek 2013.

9 Kleffner 2013, 31.

10 Ebd.

11 Vgl. Lubmann 1981.

12 Vgl. Butler 2007.

13 Um ein Beispiel zu nennen: Lange Zeit trauerten die Menschen in Europa nicht um die ertrunkenen Flüchtlinge an den europäischen Grenzen, sie wussten vielleicht nicht einmal von ihnen. Es brauchte erst eine Skandalisierung dieser Tode und nicht zuletzt einen Papstbesuch, um die Toten über Kreise von Flüchtlingsaktivisten hinaus zu betrauernswerten Menschen zu machen.

14 Vgl. Butler 2007, $15 \mathrm{ff}$.

NK 26. Jg. 2/2014 
nach der Ächtung ihrer toten Verwandten als „kriminelle Ausländer“ machen deutlich, wie solch eine Auslöschung als Verweigerung der Anrede als BürgerIn funktioniert.

Abbildung 1: „Vermisst“-Aktion des BMI „Hassan“
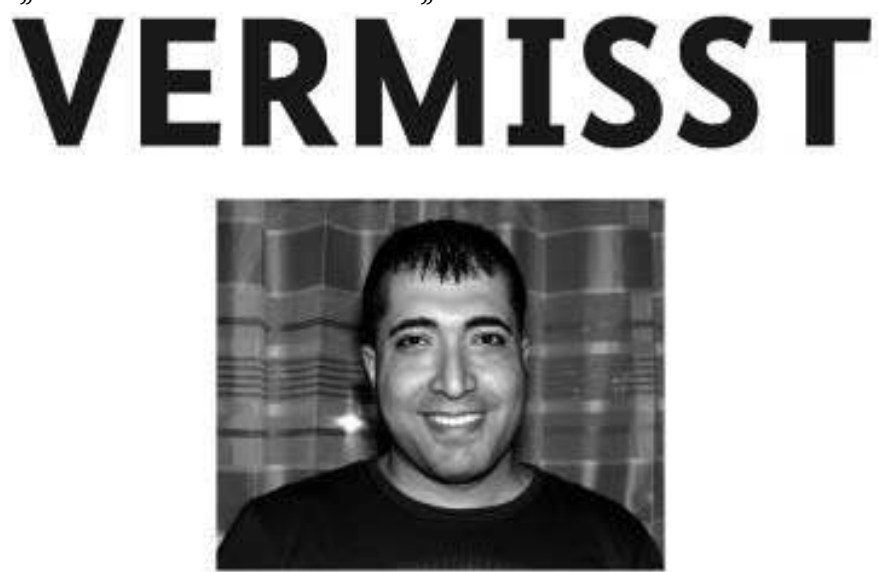

Das ist mein Bruder Hassan. Ich vermisse ihn, denn ich erkenne ihn nicht mehr. Er zieht sich immer mehr zurück und wird jeden Tag radikaler. Ich habe Angst ihn ganz zu verlieren - an religiöse Fanatiker und Terrorgruppen. Wenn es Dir so geht wie mir, wende Dich an die Beratungsstelle Radikalisierung unter 0911 - 9434343 oder beratung@bamf.bund.de Mehr Infos findest Du auf www.bamf,de/beratungsstelle

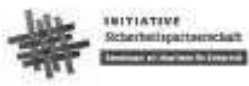

Ein weiteres prägnantes Beispiel für die Umkehr von Opfern zu Tätern stellt eine Postkartenverteilaktion - wohlgemerkt nach dem Bekanntwerden der Terrorzelle im Herbst 2012 - des Bundesministerium des Inneren in der Kölner Keupstraße dar. In dieser bunten, multikulturellen Straße im Herzen der Kölner Innenstadt hatte der NSU am 9.6.2004 ein Nagelbombenattentat verübt, bei dem 22 Menschen teilweise schwer verletzt worden sind. Direkt am Tag nach dem Attentat waren der damalige Innenminister Otto Schily und der Innenminister Nordrhein-Westfalens vor die Presse getreten und hatten einen rechten Hintergrund der Tat ausgeschlossen. Alternativ wurde nach Drogengeschäften und Schutzgelderpressungen gesucht. Die Hinweise und Sorgen der Anwohner, die schon sehr früh einen rechtsradikalen Hintergrund vermuteten, wurden ignoriert. „Dafür, dass wir der NSU-Terrorgruppe nicht früher auf die Spur gekommen sind, tragen ich und die Länderinnenminister die politische Verantwortung “, formulierte Schily im April 2012 im Tagesspiegel. ${ }^{15}$ Im September 2012 nun wurden in dieser Straße Postkarten

15 Abrufbar unter: http://www.tagesspiegel.de/politik/nsu-terror-schily-gibt-schweren-irrtumzu/6531284.html, letzter Aufruf: 26.3.14. 
mit den Bildern junger Männer und einer jungen Frau im Kopftuch verteilt, die dazu aufriefen, verdächtiges Verhalten zu melden (vgl. Abbildung 1).

Theodor W. Adorno argumentiert, dass das in der Moderne unwiederbringlich verlorene kollektive Ethos sich der Gewalt bediene, um den Schein seiner Allgemeinverbindlichkeit aufrechtzuerhalten. ${ }^{16}$ Inwiefern manifestiert sich im Sinne Adornos ein Akt solcher institutionell verübter Gewalt, wenn just in der Keupstraße das Innenministerium dazu aufruft, "islamistische Terroristen" in der Familie und im Freundeskreis aufzuspüren? Dieselben Personen, denen im Anschluss an ihre Opferwerdung kriminelle Handlungen unterstellt wurden, denen man nicht glaubte, dass Fremdenfeindlichkeit ein mögliches Tatmotiv sein könnte, werden nun vor der potentiellen Anfälligkeit ihrer Söhne, Brüder, Töchter und Freundinnen für islamischen Terrorismus gewarnt.

Melden sollen sich die Angehörigen der mutmaßlichen Terroristinnen und Terroristen beim Bundesamt für Migration und Flüchtlinge. „Diejenigen, die gesichtslos bleiben oder deren Gesichter uns als so viele Symbole des Bösen präsentiert werden, ermächtigen uns, empfindungslos zu werden bei all den Menschenleben, die wir ausgemerzt haben und deren Betrauerung unendlich aufgeschoben wird“, schreibt Butler. ${ }^{17}$ Und weiter:

„Die Entleerung des Menschlichen durch das Bild in den Medien muß dennoch unter dem Gesichtspunkt des umfassenderen Problems verstanden werden, daß normative Schemata der Intelligibilität die Etablierung dessen bewirken, was als menschlich gelten wird und was nicht, was ein lebenswertes Leben sein wird und was ein betrauernswerter Tod. Diese normativen Schemata wirken nicht bloß, indem sie Ideale des Menschlichen erzeugen, die einen Unterschied zwischen denjenigen machen, die mehr oder weniger menschlich sind. Zuweilen erzeugen sie Bilder von Untermenschen in der Vorstellung als Menschen, um zu zeigen, wie sich das Untermenschentum verstellt und diejenigen von uns zu betrügen droht, die möglicherweise glauben, in jenem Gesicht einen anderen Menschen zu erkennen. Aber manchmal funktionieren diese normativen Schemata gerade dadurch, daß sie kein Bild, keinen Namen, keine Erzählung liefern, so daß es niemals ein Leben und niemals einen Tod gegeben hat." ${ }^{18}$

Es ist also nicht unerheblich, ob und wie Menschen kollektiv betrauert werden, ob und wie sie sich als Opfer fühlen und auf die Solidarität ,der Anderen' zählen dürfen. Und dabei geht es nicht nur um Blumen, Kerzen oder Kuscheltiere, sondern auch um eine spezifische Form der Rücksichtnahme, die trauernden Menschen, wenn sie als solche erkannt werden, üblicherweise zugestanden wird. Der Fall der Opfer des NSU und ihrer Angehörigen macht deutlich, dass rassistische Stereotype nicht nur zu Ermittlungsfehlern, sondern auch dazu führten, die Betrauerbarkeit der Ermordeten und das Recht ihrer Angehörigen auf Trauer in Zweifel zu ziehen.

Neben der Figur der Täter-Opfer-Umkehr finden sich in der Berichterstattung auch zwei weitere Motive: Erstens die Artikulation des Zweifels an einem scheinbar gefestigten Opferstatus und zweitens und nicht selten damit verbunden die Einführung alter-

16 Adorno 1996, 32ff., dazu Butler 2007, $9 \mathrm{ff}$.

17 Butler 2005, 14.

18 Butler 2005, 173.

NK 26. Jg. 2/2014 
nativer Opferpositionen, die mit den vom NSU ermordeten Opfern in Konkurrenz um Aufmerksamkeit stehen. Im folgenden Fallbeispiel wird die Opferposition hinterfragt und die deutsche Nation als Opfer anderer Mechanismen, wie zum Beispiel des „Rassismus gegen Deutsche“, dem gegenüber gestellt.

Selbst nach dem Bekanntwerden der Terrorzelle ist die Rolle der Hinterbliebenen und Opfer keine sichere Sache. Ein Beispiel, das dieser Prekarität Ausdruck gibt, ist eine in der Zeitung Die Welt vom 5.3.13 gedruckte Rezension des bereits zuvor erwähnten Buchs von Semiya Şimşek. ${ }^{19}$ Die Autorin Necla Kelek steigt ein, indem sie betont, es verbiete sich angesichts Şimşeks Trauer von selbst, das Buch kritisch zu kommentieren. ${ }^{20}$ Das wäre „so passend wie ein Zwischenruf bei einer Trauerrede“. Doch schnell revidiert Kelek ihren Einstieg, begründet dies damit, dass das Buch moralisch argumentiere und daher eine politische Antwort provoziere. ${ }^{21}$ Damit wird aus der Rezension eine Anklageschrift gegen die Opferwerdung der Familie Şimşek. Und so kritisiert Kelek unter anderem die islamische Erziehung der Şimşek-Kinder und vollzieht eine ausführliche Kritik der „Islamverbände und Türkenlobby“ (sic!). ${ }^{22}$ Kelek kritisiert, dass Şimşek ihre islamische Erziehung als glücklich darstellt und sich nicht nur privat, sondern auch politisch äußere. Die Morde des NSU würden von islamischen Verbänden instrumentalisiert. ${ }^{23}$ Diese Figur ist interessant, da sie Şimşek in die Sphäre des Privaten zu bannen wünscht, die jener einer mündigen („integrierten“) Staatsbürgerin entgegensteht.

Die Kritik am Islam scheint Necla Kelek so wichtig, dass Opfern, die der islamischen Religion angehören bzw. diese nicht immanent kritisieren, der Schutz versagt wird. Das funktioniert unter anderem, indem der Islam als eigentlicher Angreifer identifiziert wird. Über Ehrenmorde werde geschwiegen, während die NSU-Morde thematisiert würden, so Kelek. ${ }^{24}$ Neben die NSU-Morde und die Trauer der Tochter werden die Opfer sogenannter Ehrenmorde gestellt und treten gleichsam in Konkurrenz. Durch die Einführung anderer, mindestens „ebensoguter" Opfer wird die autobiographische Position Şimşeks unterminiert und entpolitisiert. Kelek schreibt: „Über die moralische Betroffenheit soll Kritik am Islam und seiner Kultur wieder einmal tabuisiert und als rassistisch stigmatisiert werden. Darüber hinaus wird eine Kollektivschuld der deutschen Mehrheitsgesellschaft unterstellt.“ ${ }^{25}$ Und schließlich, so Kelek, gebe es auch „Rassismus gegen Deutsche". 26

Das Beispiel macht deutlich, wie der Ausschluss aus dem Kreise der Schutzbedürftigen argumentativ funktioniert. So macht die Rezension zwischen den Zeilen aus einer trauernden Tochter eine (Mit-)Täterin im Namen des Islam, eine Nestbeschmutzerin, eine

19 Abrufbar unter: http://www.welt.de/kultur/literarischewelt/article114130995/Semiya-Simseks-Trauer-um-ihren-ermordeten-Vater.html, letzter Aufruf: 26.3.14.

20 Kelek 2013.

21 Ebd.

22 Ebd.

23 Ebd.

24 Kelek 2013.

25 Ebd.

26 Ebd. 
schlecht integrierte Muslima die sich besser nicht öffentlich hätte äußern sollen, vor allem nicht „politisch“. Der Beitrag zeigt stellvertretend für andere Kommentare, die beispielsweise kritisieren, dass den Opferfamilien finanzielle Wiedergutmachung geleistet werde, wie die Einnahme der Opferposition den Angehörigen der Opfer noch immer verweigert wird. Die Umkehrung der Opfer zu Tätern und das Ablenken vom Thema (im Falle der Rezension Keleks in Richtung „Salafisten“, „Ehrenmorden“ oder „Rassismus gegen Deutsche") sind dabei wiederkehrende Motive der Argumentation. Zum Ende des Jahres 2013 und zu Beginn des Jahres 2014 mehrten sich auch die Berichte über die Eltern der Terroristen Uwe Mundlos und Uwe Böhnhard. Dies liegt vor allem an ihren Auftritten vor Gericht, die ausführliche Berichterstattung erfuhren. Bereits 2012 aber hatten die Eltern von Uwe Böhnhard die Öffentlichkeit gesucht und an einer 30-minütigen Dokumentation der ARD-Sendung „Panorama“ unter dem Titel „Vom Nesthäkchen zum Terroristen: Die Verzweiflung der Eltern Böhnhard“27 mitgewirkt. Auch die Berichterstattung über die Eltern der TäterInnen als potentiell weitere „Opfer“ des NSU ist ein Beispiel dafür, wie Täterschaft und Opferschaft öffentlich verhandelt werden.

\section{Der prekäre Opferstatus der NSU-Opfer. Der laufende Kampf um Deutungshoheit}

„Die Forderung nach einem wabrhaftigeren Bild, nach mebr Bildern, nach Bildern, die den ganzen Schrecken und die Wirklichkeit des Leidens übermitteln, ist wichtig und angebracht. (...) Es wäre aber ein Febler, zu glauben, wir müßten lediglich die richtigen und wabren Bilder finden, und dann werde eine bestimmte Wirklichkeit schon übermittelt. Die Wirklichkeit wird nicht von dem vermittelt, was im Bild dargestellt wird, sondern dadurch, daß die Darstellung, welche die Realität übermittelt, in Frage gestellt wird. " 28

„Und meine Mutter begann zu seufzen, zu stöbnen und zu schluchzen, sie schrie unter Tränen, mehrere Minuten lang schüttelte der Weinkrampf sie. Der Polizist hatte es letztlich doch geschafft, sie zu brechen, ibren Glauben an ibren Mann, zumindest für diesen Moment. War denn, so schoss es meiner Mutter durch den Kopf, ibr ganzes Leben eine Lüge gewesen, ibre Ehe eine Lüge, ibre Ehrbarkeit, ibr Fleiß, ibr Glaube, ibre Mekkareise, alles Lüge, Lüge, Lüge? Konnte das möglich sein, was der Polizist da bebauptete?

Nein, konnte es nicht!

Und wenn doch? "29

27 Panorama vom 19.04.12, 21:45 Uhr. Abrufbar unter: http://daserste.ndr.de/panorama/archiv/ 2012/panorama4005.html. Vgl. dazu die ausführliche Besprechung von Andreas Förster in der „Frankfurter Rundschau“ vom 19. April 2012, abrufbar unter: http://www.fr-online.de/neonazi-terror/ard-doku-ueber-nsu-mein-sohn-uwe-boehnhardt--der-moerder, 1477338,14948832.html, letzter Aufruf: 3.4.14.

28 Butler 2005, 14.

29 Simsek 2013, 121.

NK 26. Jg. 2/2014 
Bis heute kämpfen die Opfer des NSU mit der Fragilität der ihnen zugeschriebenen Rolle in der Vergangenheit und sind so noch immer sehr zurückhaltend hinsichtlich öffentlicher Äußerungen. Doch immer häufiger treten Opferangehörige und Opfer des NSUTerrors offen auf. Die Interessengemeinschaft Keupstraße veranstaltet regelmäßig Informationsabende und nimmt Einladungen an, weitere Familienangehörige von Opfern schreiben oder sprechen öffentlich über ihre Erfahrungen. Interessant, dass es ausgerechnet die Frauenzeitschrift Brigitte war, die den Opfern bereits früh ausführlichere Artikel widmete. Viele andere zogen nach. Doch weiterhin werden in Medienauftritten auch andere potentielle „Opfer" wie der Verfassungsschutz, die Bundesrepublik Deutschland und die Eltern der TerroristInnen entdeckt und ,getestet'. Andere Thematisierungen, wie jene Necla Keleks, zweifeln den Opferstatus der Familien der Opfer gezielt an, indem sie auf mögliche Verfehlungen der Opfer, auf ihre Fremdheit und das Bild des „guten Deutschlands“ pochen.

Welches vorläufige Fazit lässt sich im März 2014 aus den medialen Debatten um NSU und rassistischer Gewalt ziehen? Medial und politisch wird verhandelt, wie „gut“ sich Deutschland 2014 fühlen darf. Das Fahnenschwenken während der Weltmeisterschaft 2006 und die Führungsrolle in Europa machten mehr Lust auf derlei ,unschuldigen' Patriotismus. Über lange Zeit ungesühnte Nazimorde und ein von Konsequenzen bisher weitgehend verschonter Verfassungsschutz, dessen fragwürdigen „Unterstützungsleistungen" für RechtsterroristInnen und Rechtsextreme inzwischen deutlich herausgearbeitet wurden, stehen dem entgegen. ${ }^{30}$ Und so entsteht eine Schere zwischen der Selbstdarstellung einer guten und gereinigten Nation und der Narration einer Kontinuität rassistischer Wahnwelten, die mit tödlicher Konsequenz jene trifft, die nicht „zu uns“ gehören.

Diese Schere in der nationalen Selbstdarstellung ist für Regierung und die Parteien, die an ihr beteiligt sind, ein Problem. Denn sie haben ein Interesse, am Bild der guten Nation $\mathrm{zu}$ arbeiten und es für sich nutzbar zu machen. Nachrichten über Nazimorde sind aus dieser Perspektive keine gute PR, schon gar nicht, wenn verschiedene Ermittlungsbehörden sich dabei so gar nicht mit Ruhm bekleckert haben. Eingedenk dieser Konstellation ist es keine Überraschung, dass die Rolle des Verfassungsschutzes im NSU-Komplex bisher nicht aktiv untersucht wurde, obschon die Behörde über ihre V-Männer an der Organisation und Finanzierung der Zwickauer Zelle beteiligt war und mehrfach Beweismittel vernichtet wurden. ${ }^{31}$ Selbst das diskreditierte V-Leute-System wird nicht zur Disposition gestellt. „Die Verlautbarungen aus den Koalitionsverhandlungen von CDU und SPD, man werde ,alle 47 Empfehlungen' des Untersuchungsausschusses umsetzen, entpuppen sich bei genauerem Hinsehen an vielen Stellen vor allem als Versprechen, die komplette Wunschliste der ordnungspolitischen Horrorszenarien vieler CDU/SPD-Innenpolitiker endlich in die Tat umzusetzen: Dazu gehören vor allem mehr Geld und

30 Vgl. für viele Gensing 2012, Kleffner 2013, 34ff., Kleffner 2014; Schäfer et al. 2012 sowie Bericht des NSU-Untersuchungsausschusses des Deutschen Bundestags 2013.

31 Vgl. Kleffner 2013, 3; vgl. Bericht des NSU-Untersuchungsausschusses des Deutschen Bundestags 2013. 
Befugnisse für das Bundesamt für Verfassungsschutz (BfV) - dem der Untersuchungsausschuss im Abschlussbericht noch die Hauptverantwortung für das Versagen der Nachrichtendienste im NSU-Komplex attestiert hatte. " 32 All dies lässt auch für die Zukunft nicht auf eine Reform dieser Behörde hoffen.

Das Münchner Oberlandesgericht wird die vielen offenen Fragen, die sich zur Rolle der Geheimdienste und Ermittlungsbehörden stellen, ebenso wenig lösen können, wie die Frage danach, wie stark und wie groß das rechtsterroristische Netzwerk de facto ist. Dass es nicht auf die wenigen Menschen, die angeklagt sind, beschränkt sein wird, darauf lassen die Verhandlungsprotokolle zum Fall der Ermordung Michèle Kiesewetters ebenso schließen wie die publizistischen Aktivitäten „pro NSU“ in rechtsradikalen Magazinen.

Für die Familien und Freunde der Opfer bedeutet diese politisch-mediale Konstellation, dass ihr Opferstatus weiterhin prekär bleibt, denn sie sind keine Opfer, die sich das „gute Deutschland“ gerne vor Augen hält und betrauert. Zu schwer wiegt hier die Verantwortung politischer EntscheidungsträgerInnen und der Ermittlungsbehörden, zu viel Leid wurde den Familien bereits zugefügt. In einem Land, das an seine Läuterung glauben will, sind die Opfer des NSU und ihre Familien eine ständige Irritation, eine Störung der guten Ordnung. Die große Koalition scheint wenig Interesse daran haben und die kleine Opposition wird sich vermutlich Themen zuwenden, die mehr positive Aufmerksamkeit zu generieren versprechen. Umso wichtiger sind dann künstlerische Interventionen und die anhaltende Berichterstattung zum Thema. Eine Wanderausstellung zu den Opfern des NSU (http://www.opfer-des-nsu.de/) vermittelt bspw. Informationen über die ermordeten Menschen und den Ablauf der Ermittlungen. Die JournalistInnen von NSUWatch (http://www.nsu-watch.info/) dokumentieren den Prozess in mehreren Sprachen und stellen Hintergrundinformationen bereit. Von solchen und anderen Projekten wird vornehmlich abhängen, $o b$ und wie der NSU-Terror und seine Opfer erinnert werden.

\section{Literatur:}

Adorno (1996) Probleme der Moralphilosophie, in: Nachgelassene Schriften, Abt. IV Band 10

Bericht des NSU-Untersuchungsausschusses des Deutschen Bundestags vom 22.8.2013. Drucksache 17/14600. Abrufbar unter: http://dipbt.bundestag.de/dip21/btd/17/146/17 14600.pdf, letzter Aufruf: 3.4.14

Butler (2005) Gefährdetes Leben. Politische Essays

Butler (2007) Kritik der ethischen Gewalt 
Gensing (2012) Terror von Rechts. Die Nazimorde und das Versagen der Politik Kleffner (2013) NSU: Rassismus, Staatsversagen und die schwierige Suche nach der Wahrheit, in: Schmincke \& Siri (2013) (Hg):: NSU-Terror. Ermittlungen am rechten Abgrund, S. 29-42

Kleffner (2014) Der Bundes-NSU-Untersuchungsausschuss. Abrufbar unter: http://w ww.linksnet.de/de/artikel/30261, letzter Aufruf 3.4.14

Lubmann (1981) Politische Theorie im Wohlfahrtstaat

Schäfer, Wache E Meiborg (2012) Gutachten zum Verhalten der Thüringer Behörden und Staatsanwaltschaften bei der Verfolgung des ,Zwickauer Trios', abrufbar unter: https://www.thueringen.de/imperia/md/content/tim/veranstaltungen/120515_schaefer _gutachten.pdf, bes. S. 239ff., letzter Aufruf: 3.4.14

Schmincke \& Siri (2013) (Hg.) NSU-Terror. Ermittlungen am rechten Abgrund Simssek (2013) Schmerzliche Heimat

Kontakt:

Dr. Jasmin Siri

Ludwig-Maximilians-Universität München

Institut für Soziologie

Konradstraße 6

80801 München

jasmin.siri@soziologie.uni-muenchen.de 